\title{
Emergency department utilization during the COVID-19 pandemic
}

\author{
Regina K. Saylor, Andrea Blome, Derek Isenberg, Daniel A. DelPortal, Wayne A. Satz, Kraftin E. Schreyer \\ Department of Emergency Medicine, Temple University Hospital, Philadelphia, United States
}

Received: November 21, 2020

Accepted: December 23, 2020

Online Published: 00-00-0000

\begin{abstract}
Objective: Optimizing resource utilization is critical to reducing healthcare costs. Our study aims to review trends in overall patient volume, acuity, time of presentation, and use of resources in the emergency department (ED) during the COVID-19 pandemic.

Methods: We compared ED utilization from a 30-day period during the height of the COVID-19 pandemic (April 1, 2020-April 30,2020 ) to the same 30-day period in the preceding calendar year (April 1, 2019-April 30-2019). Data were grouped into outcome measures focusing on ED throughput and utilization of ancillary ED services.

Results: While the absolute number of patients in or arriving to the ED at any given time was significantly lower during the COVID-19 pandemic $(p<.01)$, the hourly patterns of patient census, arrivals, and admissions all aligned with pre-pandemic values. Also, patient acuity, as measured by ESI level, did not significantly change. The absolute number of admissions for both sites was similar to the pre-pandemic time period, but the percentage of patients admitted over the 30-day period increased. The absolute number of radiographic and laboratory studies ordered in the ED also changed significantly $(p<.05)$, but the hourly pattern did not.

Conclusions: Our study shows significantly lower patient volumes, increased admission rates, and no significant change in the hourly throughput of the ED. Thus, our analysis suggests that shift times should not be adjusted, nor should the number or composition of providers on each shift in academic and community ED sites during the COVID national lockdown.
\end{abstract}

Key Words: Utilization, Pandemic, COVID-19

\section{INTRODUCTION}

Emergency Department (ED) throughput is a broad concept describing how patients flow within the department. Throughput is impacted by the utilization of many variables, such as staffing, inpatient capacity, and turn-around-times for lab and imaging results. Understanding resource utilization in the ED can help reduce health care costs and optimize staffing models. ${ }^{[1]}$ In the ED, as in other healthcare settings, direct care providers and ancillary services, such as laboratory and radiology services, are key resources. ED utilization, based on patient arrivals over a 24-hour period, follows a fairly regular pattern. Arrivals begin to rise around 08:00, peak around 12:00, and remain steady until 20:00, after which volume declines until the next morning. Laboratory use and radiology use generally follow a parallel curve. ${ }^{[2,3]}$

Volume and patient acuity are the key determinants of staffing needs for physicians, advanced practice providers (APPs), and nurses. They can also be useful in determining staffing for ancillary services. The goal of a staffing model is to match

\footnotetext{
* Correspondence: Andrea Blome; Email: Andrea.Blome@ ochsner.org; Address: Department of Emergency Medicine, Temple University Hospital, Philadelphia, United States.
} 
service needs with service availability. Increased demand for services occurs when the number of patients arriving to the ED for treatment exceeds the ED resources to meet that demand. ${ }^{[4]}$ Longer patient length-of-stay (LOS) (i.e. from boarding admitted patients), prolonged turn-around-times for laboratory or imaging studies, and arrival surges can all put a strain on staffing and lead to overutilization. Patient arrival and census data by hour of the day, along with counts of laboratory and radiology orders, can be used to generate or adjust staffing models for the ED, as well as associated ancillary departments. Aligning department staffing with patient demand increases efficiency and can potentially reduce cost. $^{[4]}$

There is scant information of the effects of pandemics and natural disasters on ED operations. Kazzi et al. reported on the experience in their community emergency department after the 1994 Los Angeles Earthquake. ${ }^{[5]}$ However, most of the knowledge we have about the effects of pandemics on the ED comes from the $2009 \mathrm{H} 1 \mathrm{~N} 1$ influenza pandemic. ${ }^{[6]}$ Sugarman et al. reported on their experiences during the 2009 H1N1 pandemic which struck the United States in late summer and fall. ${ }^{[7]}$ Those authors reported that the number of visits from influenza-like illness doubled from the prior seasonal influenza period of 2008-2009 with greater than five times the volume in pediatric hospitals. They reported increased LOS and the number of patients who left without being seen, as well as insufficient ED space and shortages of personal protective equipment.

As the COVID-19 pandemic progressed in our metropolitan area, ED volumes for COVID cases were increasing, but overall ED volumes were declining, due to fewer cases of non-COVID-19 related illness presenting to the ED. According to the American Hospital Association, stay-at-home orders led to a decrease in patients seeking ED care for nonCOVID-19-related illnesses. ${ }^{[8]}$

To further study the impact of the COVID pandemic on ED utilization and the resultant implications on staffing, we compared trends in overall patient metrics and use of ancillary services during April 2020 (the height of the COVID-19 pandemic) to ED utilization in April 2019.

\section{Methods}

This study took place at two hospitals in an urban health system in Philadelphia. Study Site A is an academic tertiary care center home to a three-year emergency medicine residency program. It is a 50 bed ED that is staffed by two to four board certified emergency physicians 24 hours a day and APPs 12-14 hours per day. Study Site A serves one of the most densely populated, poorest, and most violent areas in the Commonwealth of Pennsylvania. 53\% of patients are insured by Medicaid or Medicare and $25 \%$ of patients are uninsured. Study Site B is a community site and is a 19 bed ED that is staffed by two board certified emergency physicians 24 hours a day that are supplemented by APPs 12-15 hours per day. At both study sites, APPs see only patients triaged to lower Emergency Severity Index (ESI) scores (III or below). Also, study Site A has resident coverage 24 hours a day, excluding four hours per week during conference time and one six-hour period per month during journal club. Study Site B has resident coverage 8-16 hours a day. During the pandemic, resident coverage hours stayed the same, but shift times were modified based on census data. In 2019, Study Site A had approximately 95,000 visits and Study Site B had approximately 47,000 visits.

We performed an analysis of ED utilization during the 2020 COVID pandemic using routinely collected ED metrics. Data was collected from a 30-day period during the height of the COVID-19 pandemic (April 1, 2020-April 30, 2020) and was compared to the same 30-day period in the preceding calendar year (April 1, 2019-April 30-2019). Throughput metrics reflective of ED utilization, including patient census, arrivals, and admissions were evaluated during the study periods. Overall patient acuity, as determined by the ESI level, was also included. Utilization of both laboratory and radiology resources and providers was investigated. All data was extracted from our electronic medical record (EMR) EPIC developed by Epic Systems Corporation (Verona, Wisconsin). This study was not considered human subjects research, and therefore, did not require the review of the Institutional Review Board.

\subsection{Measurements}

\subsubsection{Census by hour of day}

The ED census was defined as the total number of patients present in the ED, including those in the waiting area, those who were in-process, and those who were dispositioned, but who had not yet physically left the department. Patients were counted in the census as a data point each hour they were in the ED. We also calculated the percentage change in census by hour, referred to as the delta hourly census.

\subsubsection{Arrivals by hour of day}

Arrivals were defined as the absolute number of new patients presenting to the ED in any given specific hour. We furthermore calculated the percentage change in arrival by hour, referred to as the delta hourly arrivals.

\subsubsection{Admissions by hour of day}

Admissions were defined as the absolute number of patients admitted to any inpatient or observation setting during that 
hour of the day. The percentage change in admissions by hour, referred to as the delta hourly admissions, was additionally calculated. The monthly admission rate, defined as the total number of admissions/total arrivals, was also quantified. Sample size was determined via power analysis in which the significance level was set to 0.05 and the power was set to 0.8 .

\subsubsection{Radiographic orders by hour of day}

Orders for radiographs, computed tomography (CT), and ultrasound were counted as radiographic orders, and tracked by hour of day. Each order was counted separately, for example, radiographs of the wrist and hand would count as two separate orders, as would CT scans of the brain and cervical spine.

\subsubsection{Laboratory orders by hour of day}

All laboratory orders sent from the ED were counted towards the total number of laboratory hours and tracked by hour of day. For example, complete blood count, comprehensive metabolic panel, and troponin would be considered separate orders, despite being ordered on the same patient.

\subsubsection{Emergency Severity Index (ESI) level}

All patients presenting to the ED are triaged according to the five level Emergency Severity Index Algorithm. Patients triaged to ESI levels I and II were grouped together for ease of comparative analysis, as were patients triaged to ESI levels IV and V.

For each throughput and process metric, as well as its corresponding delta, two sample t-tests were used to compare the pre-COVID-19 time period to the COVID-19 time period, defined as percent change in absolute values per hour. By convention, significance was set at $p<.05$.

\section{Results}

Full utilization data is reported in Appendix 1 (Study Site A) and Appendix 2 (Study Site B). A summary of the statistical analysis is reported in Table 1 (Study Site A) and Table 2 (Study Site B).

\subsection{Census by hour of day}

\subsubsection{Study site $A$}

The average number of patients in the ED per hour ranged from 29.0-78.2 during the pre-COVID-19 time period and 25.1-49.8 during the COVID-19 time period. The hourly census for both time periods are displayed in Figure 1a. There was a statistically significant difference in the hourly census volumes between the two time periods $(p<.01)$. The hourly delta of the census ranged from 0.88-1.23 in the pre-COVID19 time period and from 0.91-1.15 in the COVID-19 time period. The difference in the delta values was not statistically significant $(p=.75)$.

\subsubsection{Study site B}

The average numbers of patients in the ED per hour ranged from 11.5-32.8 during the pre-COVID-19 time period and 9.5-21.4 during the COVID-19 time period. The hourly census for both time periods are displayed in Figure 1b. There was a statistically significant difference in the hourly census volumes between the two time periods $(\mathrm{p}<.01)$. The hourly delta of the census ranged from 0.87-1.27 in the pre-COVID19 time period and from 0.88-1.16 in the COVID-19 time period. The difference in the delta values was not statistically significant $(p=.79)$.

\subsection{Arrivals by hour of day 3.2.1 Study site A}

During the pre-COVID-19 time period, 8,351 patients arrived to the ED, compared to 5518 patients during the COVID-19 time period. The average numbers of hourly arrivals to the ED per hour ranged from 4.1-18.4 during the pre-COVID-19 time period and 3.7-12.0 during the COVID-19 time period. Hourly arrivals for both time periods are displayed in Figure 1c. There was a statistically significant difference in the absolute number of arrivals between the two time periods ( $p$ $<.01$ ). The delta of hourly arrivals ranged from $0.76-1.77$ in the pre-COVID-19 time period and from 0.70-1.44 in the COVID-19 time period. The difference in the delta values was not statistically significant $(p=.93)$.

\subsubsection{Study site B}

During the pre-COVID-19 time period, 3,920 patients arrived to the $\mathrm{ED}$, compared to 2384 patients during the COVID-19 time period. The average numbers of hourly arrivals to the ED per hour ranged from 2.6-9.7 during the pre-COVID-19 time period and 1.7-4.8 during the COVID-19 time period. Hourly arrivals for both time periods are displayed in Figure 1d. There was a statistically significant difference in the absolute number of arrivals between the two time periods ( $p$ $<.01)$. The delta of hourly arrivals ranged from $0.82-1.64$ in the pre-COVID-19 time period and from 0.73-1.34 in the COVID-19 time period. The difference in the delta values was not statistically significant $(p=.93)$.

\subsection{Admission rate by hour of day \\ 3.3.1 Study site A}

During the pre-COVID-19 time period, 1,798 patients were admitted from the ED, compared to 1,721 patients during the COVID-19 time period. The average numbers of hourly admissions from the ED ranged from 1.1-4.3 during the preCOVID-19 time period and 0.97-3.8 during the COVID-19 time period. Hourly admissions for both time periods are 
displayed in Figure 1e. The difference in the average number of hourly admissions between the two time periods was not statistically significant $(p=.42)$. The monthly admission rate was $21.5 \%$ during the pre-COVID-19 time period and $31.2 \%$ during the COVID-19 time period. The delta of hourly ad- missions ranged from 0.59-1.74 in the pre-COVID-19 time period and from 0.73-2.48 in the COVID-19 time period. The difference in the delta values was not statistically significant $(p=.92)$.
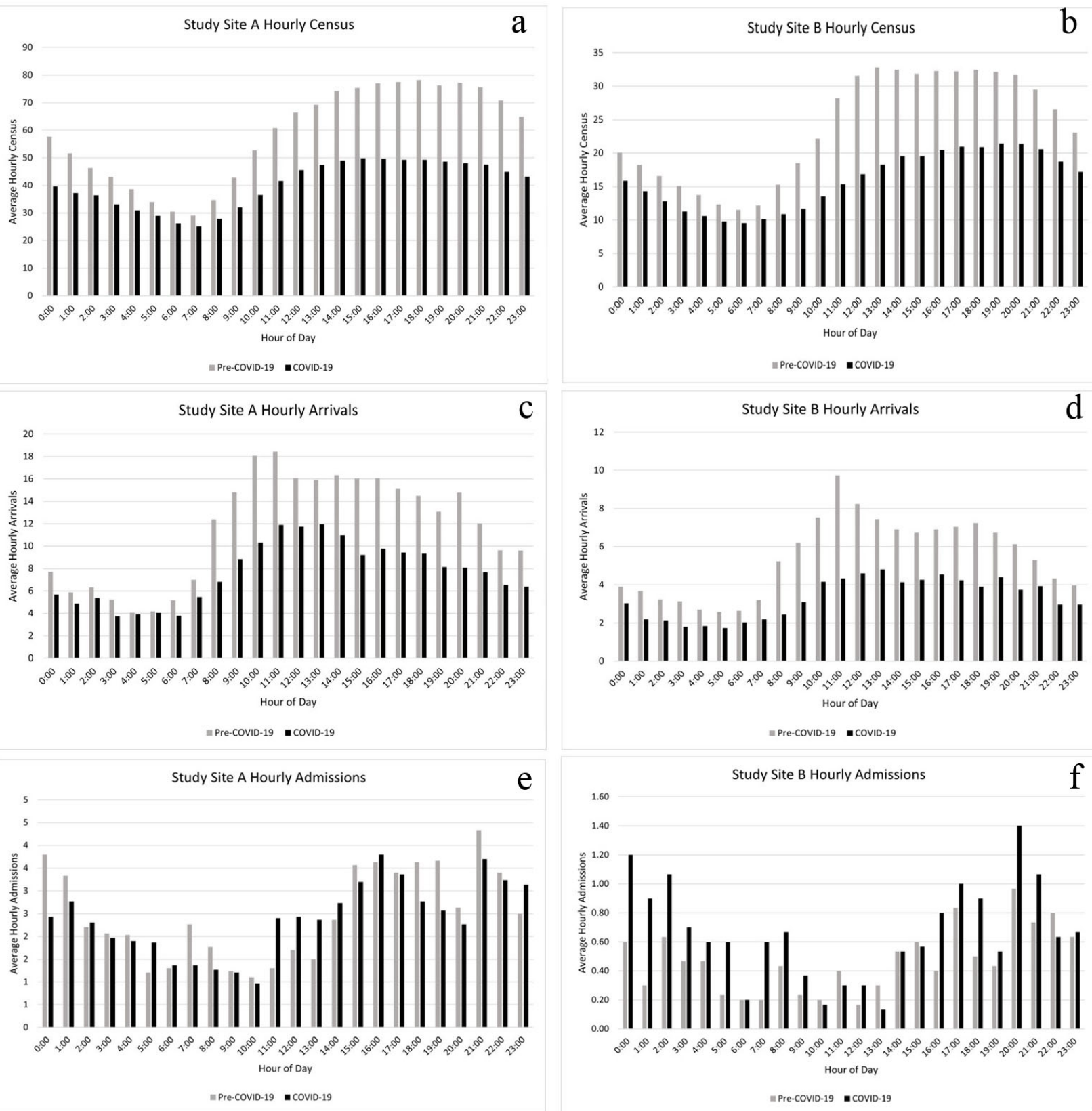

Figure 1. Throughput metrics NoteHourly census, arrivals, and admissions for study sites A and B

\subsubsection{Study site B}

During the pre-COVID-19 time period, 338 patients were admitted from the ED, compared to 477 patients during the COVID-19 time period. The average numbers of hourly admissions from the ED ranged from 0.2-1.0 during the preCOVID-19 time period and 0.1-1.4 during the COVID-19 time period. Hourly admissions for both time periods are displayed in Figure 1f. There was a statistically significant difference in the average number of hourly admissions between the two time periods $(p<.01)$. The monthly admission rate was $8.6 \%$ during the pre-COVID-19 time period and $20 \%$ during the COVID-19 time period. The delta of hourly 
admissions ranged from $0.42-2.23$ in the pre-COVID-19 time period and from $0.33-4.00$ in the COVID-19 time period. The difference in the delta values was not statistically significant $(p=.67)$.

\subsection{Radiographic orders by hour of day}

\subsubsection{Study site $A$}

During the pre-COVID-19 time period, 12,904 radiographic orders were placed in the ED, compared to 11,102 orders during the COVID-19 time period. The average numbers of radiographic orders per hour ranged from 7.9-27.0 during the pre-COVID-19 time period and 6.6-24.9 during the COVID-19 time period. Hourly radiographic orders for both time periods are displayed in Figure 2a. There was a statistically significant difference in the average absolute number of radiographic orders between the two time periods $(\mathrm{p}=0.01)$. The delta of hourly radiographic orders ranged from 0.61 1.78 in the pre-COVID-19 time period and from 0.66-1.38 in the COVID-19 time period. The difference in the delta values was not statistically significant $(p=.90)$.
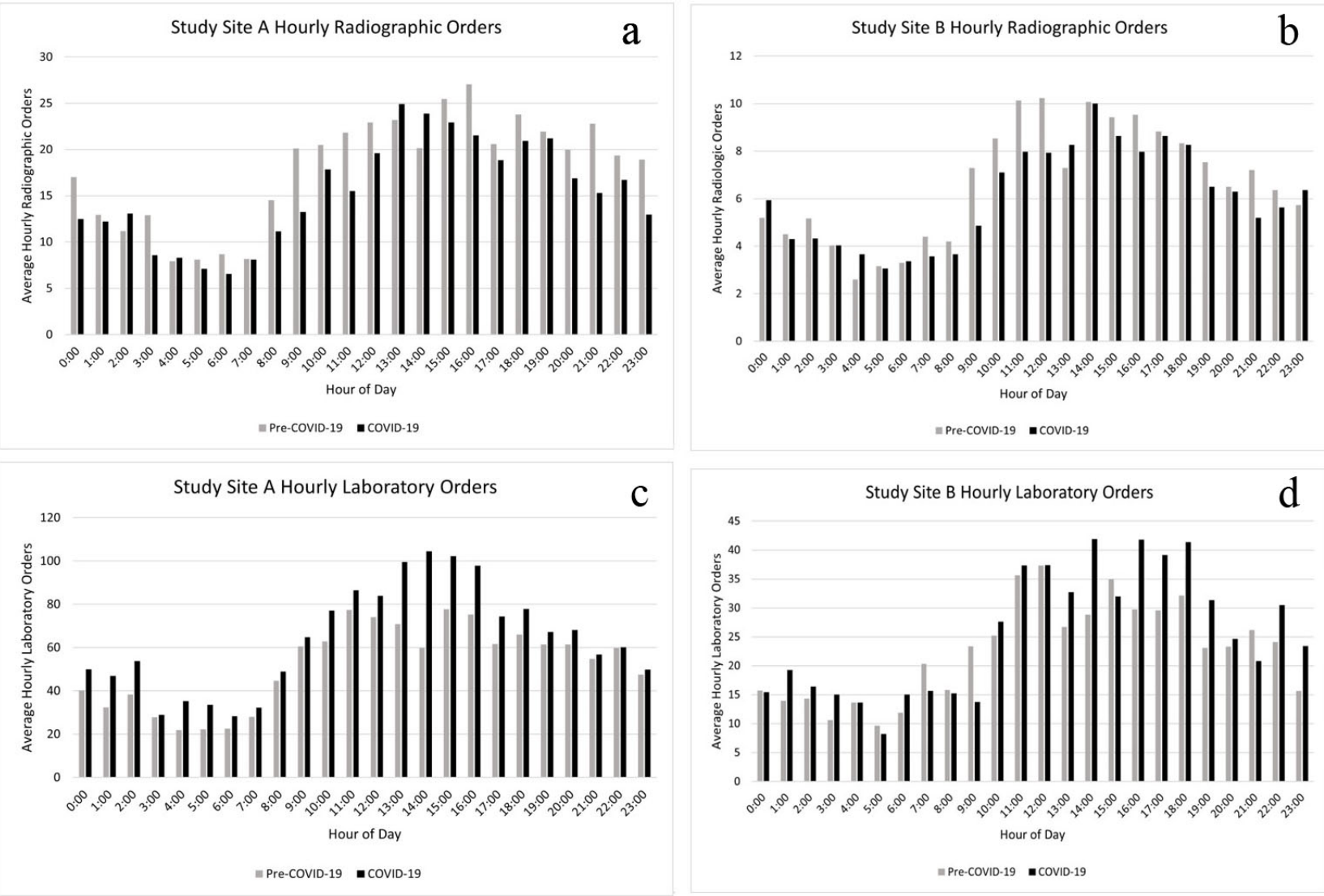

Figure 2. Utilization metrics for ancillary services at study sites A and B

\subsubsection{Study site $B$}

During the pre-COVID-19 time period, 4,788 radiographic orders were placed in the ED, compared to 4,367 orders during the COVID-19 time period. The average numbers of radiographic orders per hour ranged from 2.6-10.2 during the pre-COVID-19 time period and 3.1-10.0 during the COVID-19 time period. Hourly radiographic orders for both time periods are displayed in Figure 2b. There was a statistically significant difference in the average absolute number of radiographic orders between the two time periods $(p<01)$. The delta of hourly radiographic orders ranged from $0.64-$ 1.74 in the pre-COVID-19 time period and from $0.72-1.46$ Published by Sciedu Press in the COVID-19 time period. The difference in the delta values was not statistically significant $(p=.80)$.

\subsection{Laboratory orders by hour of day}

\subsubsection{Study site A}

During the pre-COVID-19 time period, 37,499 orders for laboratory tests were placed in the ED compared to 45,868 laboratory tests during the COVID-19 time period. The average number of laboratory orders per hour ranged from 22.0-77.7 during the pre-COVID-19 time period and 28.3-104.5 during the COVID-19 time period. Hourly laboratory orders for both time periods are displayed in Figure 2c. The difference 
in the average absolute number of laboratory orders between the two time periods was statistically significant $(p<.01)$ The delta of hourly laboratory orders ranged from $0.73-1.60$ in the pre-COVID-19 time period. In the COVID-19 time period, the range was 0.54-1.51. The difference in the delta values was not statistically significant $(p=.98)$.

\subsubsection{Study site $B$}

During the pre-COVID-19 time period, 16,259 orders for laboratory tests were placed in the ED, compared to 18,295 laboratory tests ordered during the COVID-19 time period.
The average numbers of laboratory orders per hour ranged from 9.6-37.4 during the pre-COVID-19 time period and 8.2-41.9 during the COVID-19 time period. Hourly laboratory orders for both time periods are displayed in Figure $2 \mathrm{~d}$. The difference in the average absolute number of laboratory orders between the two time periods was statistically significant $(p=.02)$. The delta of hourly laboratory orders ranged from 0.67-1.76 in the pre-COVID-19 time period. In the COVID-19 time period, the range was 0.62-2.01. The difference in the delta values was not statistically significant $(p=.80)$.

Table 1. Study site A utilization metrics

\begin{tabular}{|c|c|c|c|c|c|}
\hline Utilization Metric & Total & Range & $p$-value & Range (Delta) & $p$-value (Delta) \\
\hline \multicolumn{6}{|l|}{ Census } \\
\hline Pre-COVID-19 & 42,132 & $(29.0-78.2)$ & \multirow{2}{*}{$p<.01^{*}$} & $0.88-1.23$ & \multirow{2}{*}{$p=.75$} \\
\hline COVID-19 & 29,043 & $(25.1-49.8)$ & & $0.91-1.15$ & \\
\hline \multicolumn{6}{|l|}{ Arrivals } \\
\hline Pre-COVID-19 & 8,351 & $4.1-18.4$ & \multirow{2}{*}{$p<.01^{*}$} & $0.76-1.77$ & \multirow{2}{*}{$p=.93$} \\
\hline COVID-19 & 5,518 & $3.7-12.0$ & & $0.70-1.44$ & \\
\hline \multicolumn{6}{|l|}{ Admission } \\
\hline Pre-COVID-19 & 1,798 & $1.1-4.3$ & \multirow{2}{*}{$p=.42$} & $0.59-1.74$ & \multirow{2}{*}{$p=.92$} \\
\hline COVID-19 & 1,721 & $0.97-3.8$ & & $0.73-2.48$ & \\
\hline \multicolumn{6}{|l|}{ Radiographic } \\
\hline Pre-COVID-19 & 12,904 & $7.9-27.0$ & \multirow{2}{*}{$p=.01^{*}$} & $0.61-1.78$ & \multirow{2}{*}{$p=.90$} \\
\hline COVID-19 & 11,102 & $6.6-24.9$ & & $0.66-1.38$ & \\
\hline \multicolumn{6}{|l|}{ Laboratory } \\
\hline Pre-COVID-19 & 37,499 & $22.0-77.7$ & \multirow{2}{*}{$p<.01^{*}$} & $0.73-1.60$ & \multirow{2}{*}{$p=.98$} \\
\hline COVID-19 & 45,868 & 28.3-104.5 & & $0.54-1.51$ & \\
\hline
\end{tabular}

Note Total $=$ the total count of each individual data point collected during the 30-day time periods; Range $=$ the minimum and maximum values of the average hourly values for each metric; Delta range $=$ the minimum and maximum values of the percent hourly change for each metric; ${ }^{*}$ indicates statistical significance
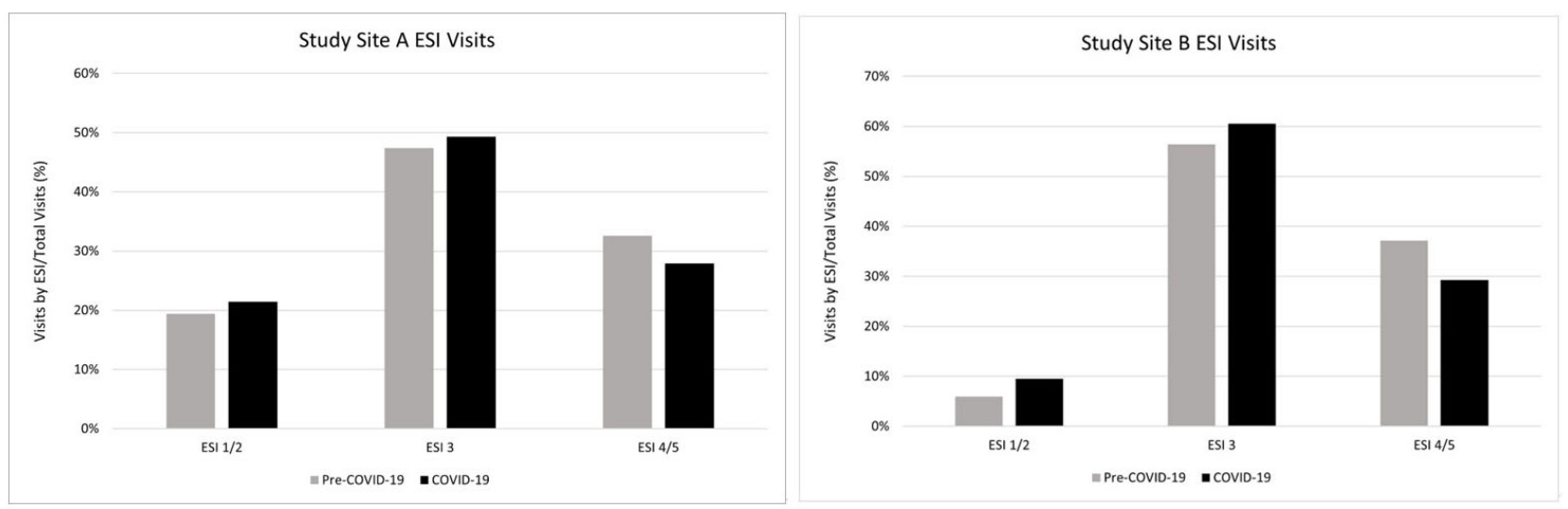

Figure 3. Patient acuity by ESI level for study sites A and B 
Table 2. Study site B utilization metrics

\begin{tabular}{|c|c|c|c|c|c|}
\hline Utilization Metric & Total & Range & $p$-value & Range (Delta) & $p$-value (Delta) \\
\hline \multicolumn{6}{|l|}{ Census } \\
\hline Pre-COVID-19 & 17,147 & $11.5-32.8$ & \multirow{2}{*}{$p<.01^{*}$} & $0.87-1.27$ & \multirow{2}{*}{$\mathrm{p}=.79$} \\
\hline COVID-19 & 11,447 & $9.5-21.4$ & & $0.88-1.16$ & \\
\hline \multicolumn{6}{|l|}{ Arrivals } \\
\hline Pre-COVID-19 & 3,920 & $2.6-9.7$ & \multirow{2}{*}{$p<.01^{*}$} & $0.82-1.64$ & \multirow{2}{*}{$\mathrm{p}=.93$} \\
\hline COVID-19 & 2,384 & $1.7-4.8$ & & $0.73-1.34$ & \\
\hline \multicolumn{6}{|l|}{ Admission } \\
\hline Pre-COVID-19 & 338 & $0.2-1.0$ & \multirow{2}{*}{$p<.01^{*}$} & $0.42-2.23$ & \multirow{2}{*}{$\mathrm{p}=.67$} \\
\hline COVID-19 & 477 & $0.1-1.4$ & & $0.33-4.00$ & \\
\hline \multicolumn{6}{|l|}{ Radiographic } \\
\hline Pre-COVID-19 & 4,788 & $2.6-10.2$ & \multirow{2}{*}{$p<.01^{*}$} & $0.64-1.74$ & \multirow{2}{*}{$\mathrm{p}=.80$} \\
\hline COVID-19 & 4,367 & $3.1-10.0$ & & $0.72-1.46$ & \\
\hline \multicolumn{6}{|l|}{ Laboratory } \\
\hline Pre-COVID-19 & 16,259 & $9.6-37.4$ & \multirow{2}{*}{$p<.02^{*}$} & $0.67-1.76$ & \multirow{2}{*}{$\mathrm{p}=.80$} \\
\hline COVID-19 & 18,295 & $8.2-41.9$ & & $0.62-2.01$ & \\
\hline
\end{tabular}

Note . Total $=$ the total count of each individual data point collected during the 30-day time periods; Range $=$ the minimum and maximum values of the average hourly values for each metric; Delta range $=$ the minimum and maximum values of the percent hourly change for each metric; ${ }^{*}$ indicates statistical significance

\subsection{Emergency Severity Index (ESI) level}

\subsubsection{Study site $A$}

During the pre-COVID-19 time period, $19.4 \%$ of total visits were patients triaged to ESI level I or II, compared to $21.4 \%$ of patients during the COVID-19 time period. $47.4 \%$ of patients were triaged to ESI level III in the pre-COVID-19 time period, compared to $49.4 \%$ during the COVID-19 time period. During the pre-COVID-19 time period, $32.6 \%$ of patients were triaged to either ESI level IV or V, compared to $27.9 \%$ of patients during the COVID-19 time period. There was no statistically significant difference in the composition of patient acuity $(p=.92)$. ESI visits for Study Site A are shown in Figure 3a. Given the difference in volume between Study Sites A and B, ESI I/II and III/IV were combined in Figure 3a.

\subsubsection{Study site B}

During the pre-COVID-19 time period, $5.9 \%$ of total visits were patients triaged to ESI level I or II, compared to $9.5 \%$ of patients during the COVID-19 time period. 56.4\% of patients were triaged to ESI level III in the pre-COVID-19 time period, compared to $60.6 \%$ during the COVID-19 time period. During the pre-COVID-19 time period, $37.2 \%$ of patients were triaged to either ESI level IV or V, compared to $29.2 \%$ of patients during the COVID-19 time period. There was no statistically significant difference in the composition of patient acuity $(p=.99)$. ESI visits for Study Site B are shown in Figure $3 b$.

\section{Discussion}

This study analyzed the utilization and throughput metrics of two urban EDs during the height of the COVID-19 pandemic and compared those metrics to the prior year. In doing so, we derived trends that could be used to predict staffing needs in times of anticipated patient surges. Additionally, by using both Study Site A and Study Site B, our analysis may be representative of both academic tertiary care sites and smaller urban community sites.

We found that the absolute number of patients in or arriving to the ED at any given time was significantly lower during the COVID-19 pandemic. Conversely, the absolute number of admissions for both sites was similar to the pre-pandemic time period. Accordingly, the percentage of patients admitted over the 30-day period increased. Despite these differences, the hourly patterns of patient census, arrivals, and admissions all remained statistically similar to pre-pandemic values. In short, though there were significant changes in patient volume and admission rates, we found no significant change in the hourly throughput of the ED.

As demonstrated, the patient census significantly decreased during the height of the pandemic. Similarly, ED volumes decreased 50\% during the height of the COVID pandemic. ${ }^{[9]}$ Although the total percentage of COVID and influenza-like illness tripled, total ED visits were down over 1,000,000 visits per week during the height of the epidemic. Similar decreases were found in England after their country's 
lockdown. ${ }^{[10]}$ This phenomenon has not been documented in previous pandemics and may be attributed to the stay-athome order enacted for COVID-19. ED visits were down $25 \%$ during the week after the lockdown and $49 \%$ from the month before. It also may be attributed to patient desire to avoid potential exposure to the virus.

Given the statistically significant difference in absolute patient volumes, it might be argued that overall staffing could be adjusted to mimic predicted trends and potentially reduce staff exposure in a future pandemic. For example, the absolute number of providers on each shift could be reduced, or overlapping provider shift hours could be eliminated. However, it is important to consider the notion of staff reduction in relation to the fact that the overall admission rates of patients significantly increased at both our tertiary academic and community sites. Additionally, the hourly census delta did not change. This is important as the hourly census delta is a reasonable reflection of the state of the ED over time, i.e. it accounts not only for patient arrivals but also for throughput issues such as LOS. The lack of difference in the census delta may be explained by a number of factors, such as increased LOS due to staff donning and doffing of PPE, delays for inpatient beds requiring isolation, or evolving admission algorithms. Regardless of the explanation, there was no significant change in the hourly census delta nor hourly arrivals delta, and thus, it could be reasoned that neither a reduction in staff nor a change to shift hours would likely produce a meaningful difference in ED throughput or patient care.

In addition to the absolute number of providers available in the ED, appropriate staffing also relies upon the ratio of physicians to APPs. This ratio is often based upon the expected level of patient acuity in the ED at a given time. During the COVID-19 pandemic, both study sites saw a slightly higher patient acuity (70.8\% of patients at Study Site A were ESI 3 or higher vs $66.8 \%$ pre-pandemic, $70.1 \%$ vs $62.3 \%$ at Study Site B). However, this difference was not statistically significant. Accordingly, the findings do not imply a need to adjust the ratio of physicians to APPs.

Regarding ED utilization of ancillary services, our analysis found that while the absolute number of patients in either department decreased during the COVID-19 pandemic, the absolute number of laboratory tests was significantly higher at both study sites. This finding could potentially be explained by the number of investigative labs sent in the ED to facilitate inpatient treatment plans for COVID-19. Our site mandated approximately 20 blood tests for all patients being admitted for COVID, many of which would not typically be sent from the ED. If patient disposition depends on certain lab results, the increase in laboratory studies ordered could have important implications for patient throughput, depend- ing on the capacity of a site's laboratory. If the anticipated increase in tests exceeds the capacity of the current staffing model, the laboratory department should increase staffing during the pandemic.

Conversely, the absolute number of radiographic orders placed in both departments decreased during the COVID19 pandemic. This finding could imply that the radiology department may benefit from decreased staffing. It should be considered that while the absolute number of radiographs ordered went down, the decrease was not as significant as the decrease in total patient census. This could be explained by a greater number of orders per patient, for example a chest radiograph and CT for respiratory complaints, or by a larger percentage of patients receiving imaging. Additional studies into staffing implications for radiological services could be warranted.

Lastly, when comparing Study Site A, an academic tertiary care site, and Study Site B, a community ED, there was no significant difference in any utilization or throughput metrics. Both sites saw a decrease in overall patient volumes but interestingly saw approximately a ten-percent increase in monthly admissions. There are several potential explanations for this finding. While the sites are different, they are located in relatively close proximity to one another. Therefore, one might consider their location, and therefore, rate of viral spread very similar. The patient population also comprises very similar demographic groups. Finally, as Study Site B is under the umbrella of the same health system as Study Site A, the admission guidance was the same for both clinical sites.

Regardless, if both academic and community sites can expect sustained rates of admission during pandemics, there could be important implications for departmental throughput. A consistent admission rate during a pandemic in which those afflicted have longer inpatient LOS could lead to increased boarding in EDs and negatively impact ED flow.

\section{Limitations}

Several limitations exist for the study. First, the study was completed in an urban setting with patients predominantly of a low socioeconomic status. Because the impact and severity of the everchanging pandemic on different hospitals is difficult to predict, the results may not be generalizable to all hospital settings or patient populations. Variations in patient metrics occur at a different times/seasons of the year. Additionally, ED throughput can depend on many site-specific variables, such as inpatient capacity, which may also make the findings less generalizable. Lastly, the COVID-19 pandemic was unique in that stay-at-home orders were enacted. 
The impact on ED volume and utilization may not be generalizable to other pandemics, or future waves of COVID-19, if stay-at-home orders are not in place.

\section{Conclusions}

The COVID-19 pandemic presented a unique opportunity to investigate changes in ED utilization in order to assess the need for adjustment to staffing models. Our analysis sug- gests that overall staffing and shift times for nurses, APPS, and physicians should not be adjusted during pandemics. In addition, the ratio of APPs to physicians should not change. Similar ED utilization was seen in both urban academic and urban community clinical settings.

\section{Conflicts of InTERest Disclosure}

The authors declare they have no conflicts of interest.

\section{REFERENCES}

[1] Aledhaim A, Walker A, Vesselinov R, et al. Resource Utilization in Non-Academic Emergency Departments with Advanced Practice Providers. West J Emerg Med. 2019; 20(4). PMid: 31316691. https://doi.org/10.5811/westjem. 2019.5.42465

[2] Welch SJ, Jones SS, Allen T. Mapping the 24-Hour Emergency Department Cycle to Improve Patient Flow. Jt Comm J Qual Patient Saf. 2007; 33(5): 247-55. https ://doi .org/10.1016/S1553-7 250 (07) 33029-8

[3] Tiwari Y, Goel S, Singh A. Arrival time pattern and waiting time distribution of patients in the emergency outpatient department of a tertiary level health care institution of North India. J Emerg Trauma Shock. 2014; 7(3): 160-5. PMid: 25114424. https ://doi .org/10 .4103/0974-2700.136855

[4] Skinner J, et al. Using Predictive Analytics to Align ED Staffing Resources With Patient Demand [Internet]. HFMA. [cited 2020 May 21]. Available from: https://www.hfma.org/topics/hfm/20 18/february/59165.html

[5] Kazzi AA, Langdorf MI, Handly N, et al. Earthquake Epidemiology: The 1994 Los Angeles Earthquake Emergency Department Experience at a Community Hospital. 2000; 15(1): 20-7. https: //doi.org/10.1017/S1049023X00024882
[6] Novel Swine-Origin Influenza A (H1N1) Virus Investigation Team. Emergence of a Novel Swine-Origin Influenza A (H1N1) Virus in Humans. N Engl J Med. 2009; 360(25): 2605-15. PMid: 19423869. https://doi.org/10.1056/NEJMoa0903810

[7] Sugerman D, Nadeau KH, Lafond K, et al. A survey of emergency department 2009 pandemic influenza A (H1N1) surge preparednessAtlanta, Georgia, July-October 2009. Clin Infect Dis Off Publ Infect Dis Soc Am. 2011; 52(Suppl 1): S177-182. PMid: 21342892. https://doi.org/10.1093/cid/ciq035

[8] US hospitals are seeing fewer patients without Covid-19. (And that could be a problem). [cited 2020 May 21]. Available from: http://www.advisory.com/daily-briefing/2020/0 4/22/lost-patients

[9] Wong LE, Hawkins JE, Langness S, et al. Where Are All the Patients? Addressing Covid-19 Fear to Encourage Sick Patients to Seek Emergency Care. NEJM Catal Innov Care Deliv. 2020 [cited 2020 May 21]. Available from: https://catalyst.nejm.org/ doi/full/10.1056/CAT.20.0193

[10] Thornton J. Covid-19: A\&E visits in England fall by $25 \%$ in week after lockdown. BMJ. 2020; 369. PMid: 32253175. https: //doi.org/10.1136/bmj.m1401 